\title{
Composition, Reactivity and Regulation of Extracellular Metal-Reducing Structures (Bacterial Nanowires) Produced by Dissimilatory Metal - Reducing Bacteria
}

\author{
Beveridge, Terrance $\mathrm{J}$. \\ University of Guelph
}

\begin{abstract}
RESULTS TO DATE: Task\# 1- Contribution of LPS to Shewanella surface physicochemistry and Feoxide adherence
\end{abstract}

Approach. Previously, using conventional and cryoTEM techniques, surface physicochemistry assays, NMR structural analysis, etc., we showed that the structure and composition of Shewanella?s lipopolysaccharide (LPS) and capsular polysaccharide (PS) significantly determined overall cell surface physicochemistry. In our study a strong correlation between such macroscopic parameters as surface electronegativity, hydrophobicity or hydrophilicity, and bacterial adhesion to hematite was observed. Rough LPS strains exhibited more than an order higher affinity and maximal sorption capacity to hematite when compared to encapsulated strains. These general trends, however, characterise bacterial adhesion only as a bulk process, being unable to reveal finer mechanisms taking place at the level of an individual cell. Cell surface physicochemical and structural heterogeneity suggests much more ccomplex interactions at the bacterial-mineral interface than predicted by such approaches operating within macroscopic parameters. To overcome these limitations we extended our studies of Shewanella adhesion to a more detailed level using atomic force microscopy (AFM). This method allowed direct observation of (partially or fully) hydrated bacteria adhered to different substrata. It was also possible to probe cell surface physicochemistry at nanoscale-levels and to perform quantitative measurements of adhesion forces. We used thin films $(\sim 40 \mathrm{~nm})$ of polycrystalline Fe-oxide deposited on glass or quartz as a model of a ?pristine? mineral surface. However, in natural environments at circumneutral $\mathrm{pH}$, the surface charge of Fe-oxides can vary from positive to negative, being largely determined by adsorbed inorganic or organic ions (e.g., humics). To obtain a clearer picture of how mineral surface physicochemistry influences DMRB adhesion, we also used a wide range of negatively and positively charged surfaces with different degrees of wettability.

Progress. Systematic AFM studies of DMRB (both Shewanella and Geobacter) on model substrates displayed varying degree of phenotype heterogeneity in terms of cell surface structure and composition; rough and smooth LPS cells were present within the same population. Even S.putrefaciens CN32, which appeared ?rough? on the basis of TEM and LPS/PS analyses, possessed a small fraction of encapsulated cells, and in smooth strains (S. oneidensis MR-4) a small number of cells were rough. Furthermore, substrate physicochemistry determined selectivity towards a certain phenotype. Rough phenotypes adhered better to positively charged (e.g., Fe-oxide) and hydrophobic surfaces (C-14, Formvar), while smooth phenotypes adhered better to hydrophilic surfaces (mica, glass). Moreover, in case of overall smooth populations (MR-4 or S. algae BrYFC), only rough cells adhered to the surface of Fe-oxide. Such phenotypic plasticity, where a certain number of adhesive cells are always present, ensures an ecological advantage for DMRB in versatile subsurface environments. These and our previous physicochemical results help explain preferential adhesion by DMRB to certain surfaces.

\section{Task\# 2 - Bacterial Nanowires}

Approach and Progress. Nanowires are extremely delicate thin fibrous structures that are easily destroyed by small shear forces during their attempted isolation. There small size requires some sort of microscopy for their detection and their high resolution requires transmission electron microscopy (TEM). Preparation for TEM is therefore difficult since centrifugations, drying and staining are required, each subjecting these delicate structures to shear. For this reason, great care and experience is required for nanowire visualization and characterization. S. oneidensis MR-1 cells were grown in a fermentor under 
O2-limitation (with no other electron acceptor in the medium) with a working volume of $1 \mathrm{~L}$ for optimal production of nanowires. The fermentor was fed with CDM medium with lactate as a sole carbon source at a dilution rate of $0.1 \mathrm{~h}-1 ; \mathrm{pH}$ was maintained at 6.86 and temperature at $260 \mathrm{C}$. To reduce shear and to preserve the delicate nanowires, the agitation rate was set at $50 \mathrm{rpm}$. Both TEM and AFM were used for their detection. For AFM imaging, bacteria were deposited on different substrata and showed numerous thin structures extending from the cell. Also around each cell, exopolymeric substances (EPS) and membrane vesicles (MVs) were detected. The thin structures were thought to be nanowires and prompted a more extensive study by TEM. Cells were carefully layered onto Formvar carbon-coated copper TEM grids and stained with uranyl acetate. High resolution TEM showed the same thin nanowires that AFM did; here, they resembled thin pliable pili and often aggregated together as fibrous clumps. Intermolecular associations within the fibres could not be seen. Frequently, MVs were seen associated with the nanowires. In an attempt to characterize the composition of the fibres, immunogold labeling was attempted using a battery of antibodies (Abs) supplied by our PNNL collaborators. Here, distinct Abs against specific surface or periplasmic proteins were reacted with the specimen and anti-rabbit gold Abs used to secondarily label the proteins. These experiments revealed that the nanowires possessed distinct epitopes that had reducing power (e.g., MtrC) and similarity to type II secretion machinery (e.g., OmcA and GspG). These results support the concept that nanowires could be capable of electron transfer and could be derived from type II secretion systems. Work continues and we expect to attempt frozen-foil cryoTEM soon.

DELIVERABLES: Publications

S. Glasauer, S. Langley and T. J. Beveridge. 2004. Intracellular manganese deposits formed by a subsurface bacterium. Environmental Microbiology. (In press).

E. Vinogradov, A. Korenevsky and T.J. Beveridge. 2004. The structure of the core region of the lipopolysaccharide from Shewanella algae BrY, containing 8-amino-3,8-dideoxy-D-manno-oct-2-ulosonic acid. Carbohydrate Research 339 (3): 737-740.

E. Vinogradov, A. Korenevsky and T.J. Beveridge. 2003. The structure of the rough-type lipopolysaccharide from Shewanella oneidensis MR-1, containing 8-amino-8-deoxy-Kdo and an openchain form of 2-acetamido-2-deoxy-D-galactose. Carbohydrate Research 338 (19): 1991-1997.

\section{Conference Proceedings}

T.J. Beveridge. 2004. Those crystalline mineral garments bacteria sometimes wear (keynote speaker). Water-Rock Interaction 11th International Symposium, June 27-July2, Saratoga Springs New York, USA.

S. Glasauer. 2004. The interactions of Shewanella spp. with Fe(III) oxides (keynote speaker). 10th International Symposium on Microbial Ecology, August 22-August 27, Cancun, Mexico. S. Glasauer, S. Langley, T. J. Beveridge, M. I. Boyanov, B. Lai and K. M. Kemner. 2004 Red beans: intra- and extracellular Fe minerals formed by S. putrefaciens (invited speaker). Water-Rock Interaction 11th International Symposium, June 27-July 2, Saratoga Springs, New York, U.S.A.

S. Glasauer, J.R. Lloyd, J.C. Renshaw, A.U. Gehring, and T.J. Beveridge. 2003. Immobilization of metals at the cell wall of subsurface bacteria during anaerobic respiration (invited speaker). V. M. Goldschmidt Conference Sept. 7-12, 2003, Kurashiki, Japan.

S. Glasauer and T.J. Beveridge. The dissimilatory reduction of Mn by Shewanella putrefaciens: mineralogical and biological constraints (poster). International Conference on Environmental Biogeochemistry (ISEB 16). Oirase in Towada Hachimantai National Park, Japan. Sept. 1-6, 2003. 
V.R. Phoenix, A.A. Korenevsky, T.J. Beveridge, Y.A.Gorby and F.G. Ferris. 2004. Lipopolysaccharide and surface proton binding characterization of Shewanella sp. (invited speaker) V. M. Goldschmidt Conference June 6-11, 2004, Copenhagen, Denmark.

A. Korenevsky , Y. Gorby , T.J. Beveridge. 2004. Shewanella?s surface physicochemistry and adhesiveness to Fe-oxides. V. M. Goldschmidt Conference June 6-11, 2004, Copenhagen, Denmark. 